\title{
ADMINISTRATION OF FRUCTOSE 1,6-DIPHOSPHATE DURING EARLY REPERFUSION SIGNIFICANTLY IMPROVES RECOVERY OF CONTRACTILE FUNCTION IN THE POSTISCHEIMIC HEART
}

Koh Takeuchi, MD

Hung Cao-Danh, $\mathrm{PhD}^{\mathrm{a}}$

Ingeborg Friehs, $\mathrm{MD}^{\mathrm{a}}$

Paul Glynn, $\mathrm{PhD}^{\mathrm{a}}$

Donato D'Agostino, MD ${ }^{\mathrm{a}}$

Elena Simplaceanu, $\mathrm{MS}^{\mathrm{c}}$

Francis X. McGowan, $\mathrm{MD}^{\mathrm{b}}$

Pedro J. del Nido, MD ${ }^{a}$

\begin{abstract}
Objectives: Fructose-1,6-diphosphate is a glycolytic intermediate that has been shown experimentally to cross the cell membrane and lead to increased glycolytic flux. Because glycolysis is an important energy source for myocardium during early reperfusion, we sought to determine the effects of fructose-1,6-diphosphate on recovery of postischemic contractile function. Methods: Langendorff-perfused rabbit hearts were infused with fructose-1,6-diphosphate ( 5 and $10 \mathrm{mmol} / \mathrm{L}, n=5$ per group) in a nonischemic model. In a second group of hearts subjected to 35 minutes of ischemia at $37^{\circ} \mathrm{C}$ followed by reperfusion ( $n=6$ per group), a $5 \mathrm{mmol} / \mathrm{L}$ concentration of fructose-1,6-diphosphate was infused during the first $\mathbf{3 0}$ minutes of reperfusion. We measured contractile function, glucose uptake, lactate production, and adenosine triphosphate and phosphocreatine levels by phosphorus 31-nuclear magnetic resonance spectroscopy. Results: In the nonischemic hearts, fructose-1,6-diphosphate resulted in a dose-dependent increase in glucose uptake, adenosine triphosphate, phosphocreatine, and inorganic phosphate levels. During the infusion of fructose-1,6-diphosphate, developed pressure and extracellular calcium levels decreased. Developed pressure was restored to near control values by normalizing extracellular calcium. In the ischemia/reperfusion model, after 60 minutes of reperfusion the hearts that received fructose-1,6-diphosphate during the first 30 minutes of reperfusion had higher developed pressures $(83 \pm 2$ vs $70 \pm 4 \mathrm{~mm} \mathrm{Hg}, p<0.05)$, lower diastolic pressures $(7 \pm 1$ vs $12 \pm 2 \mathrm{~mm}$ $\mathrm{Hg}, \boldsymbol{p}<0.05)$, and higher phosphocreatine levels than control untreated hearts. Glucose uptake was also greater after ischemia in the hearts treated with fructose-1,6-diphosphate. Conclusions: We conclude that fructose-1,6diphosphate, when given during early reperfusion, significantly improves recovery of both diastolic and systolic function in association with increased glucose uptake and higher phosphocreatine levels during reperfusion. (J Thorac Cardiovasc Surg 1998;116:335-43)
\end{abstract}

$\mathrm{F}_{\mathrm{in}}^{\mathrm{r}}$ uctose 1,6-diphosphate (FDP) is a glycolytic intermediate that has been proposed as an effective drug to improve myocardial function in chronic

From the Departments of Cardiac Surgery ${ }^{\mathrm{a}}$ and Anesthesiology, ${ }^{\mathrm{b}}$ Children's Hospital and Harvard Medical School, and the NMR for Biologic Research, ${ }^{\mathrm{c}}$ Carnegie-Mellon University, Boston, Mass.

Received for publication August 19, 1997; revisions requested Dec. 15, 1997; revisions received Feb. 13, 1998; accepted for publication March 25, 1998.

Address for reprints: Pedro J. del Nido, MD, Department of Cardiac Surgery, Children's Hospital, 300 Longwood Ave., Boston, MA 02115.

Copyright (C) 1998 by Mosby, Inc.

$0022-5223 / 98 \$ 5.00+0 \quad \mathbf{1 2 / 1 / 9 0 6 7 2}$ congestive heart failure ${ }^{1}$ and in the treatment of patients in the early phase of acute myocardial infarction. $^{2}$ The exact mechanism(s) of action of FDP in these two clinical conditions is not known. However, two major effects on myocardium have been proposed to explain the ameliorative effects of FDP that may significantly affect reperfused myocardium: (1) the positive effects of FDP on glycolysis and the energetic state of the heart and (2) the ability of FDP to bind free calcium.

Although FDP is a polar molecule because of its phosphate moieties, there is now good evidence that it can cross the cell membrane in a dose-dependent manner. Hardin and Roberts, ${ }^{3}$ using carbon $13-$ nuclear magnetic resonance (NMR), demonstrated 
a.

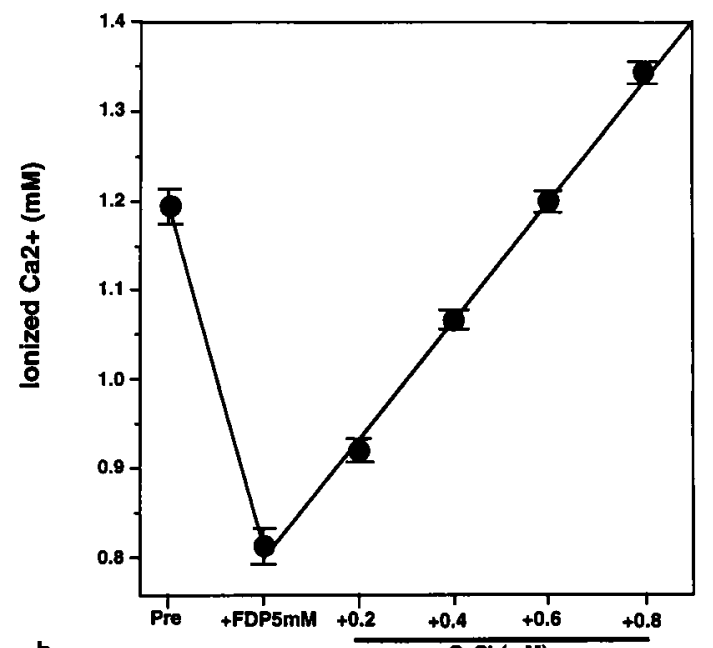

b.

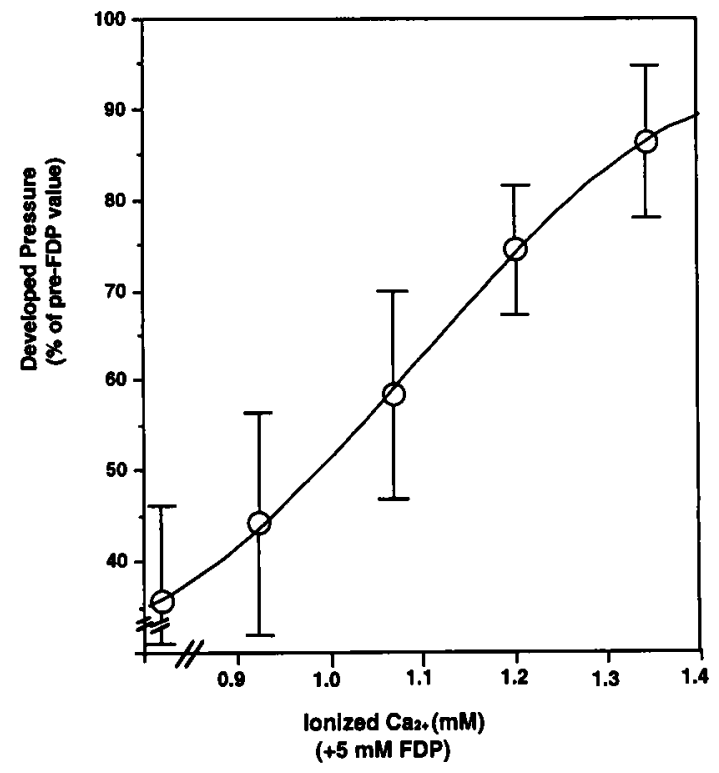

Fig. 1. a, Ionized calcium concentration in a solution of Krebs buffer alone (pre), Krebs + a $5 \mathrm{mmol} / \mathrm{L}$ concentration of FDP $(+F D P 5 m M)$, and Krebs + FDP + increasing concentrations of total calcium achieved by the addition of calcium chloride in vitro $\left(+\mathrm{CaCl}_{2}[\mathrm{mM}]\right)$. b, Developed pressure (expressed as percent of pre-FDP infusion value) in a nonischemic heart perfused with Krebs + a $5 \mathrm{mmol} / \mathrm{L}$ concentration of FDP (value at left) and Krebs + FDP + increasing ionized calcium concentrations in the perfusate (achieved by the addition of calcium chloride). All values are mean \pm standard error, $n=5$.

that exogenous ${ }^{13} \mathrm{C}$-labeled FDP can enter the cell and, during hypoxia, can lead to the generation of ${ }^{13}$ C-lactate. The ability of FDP to increase myocardial high-energy phosphates has been ascribed to
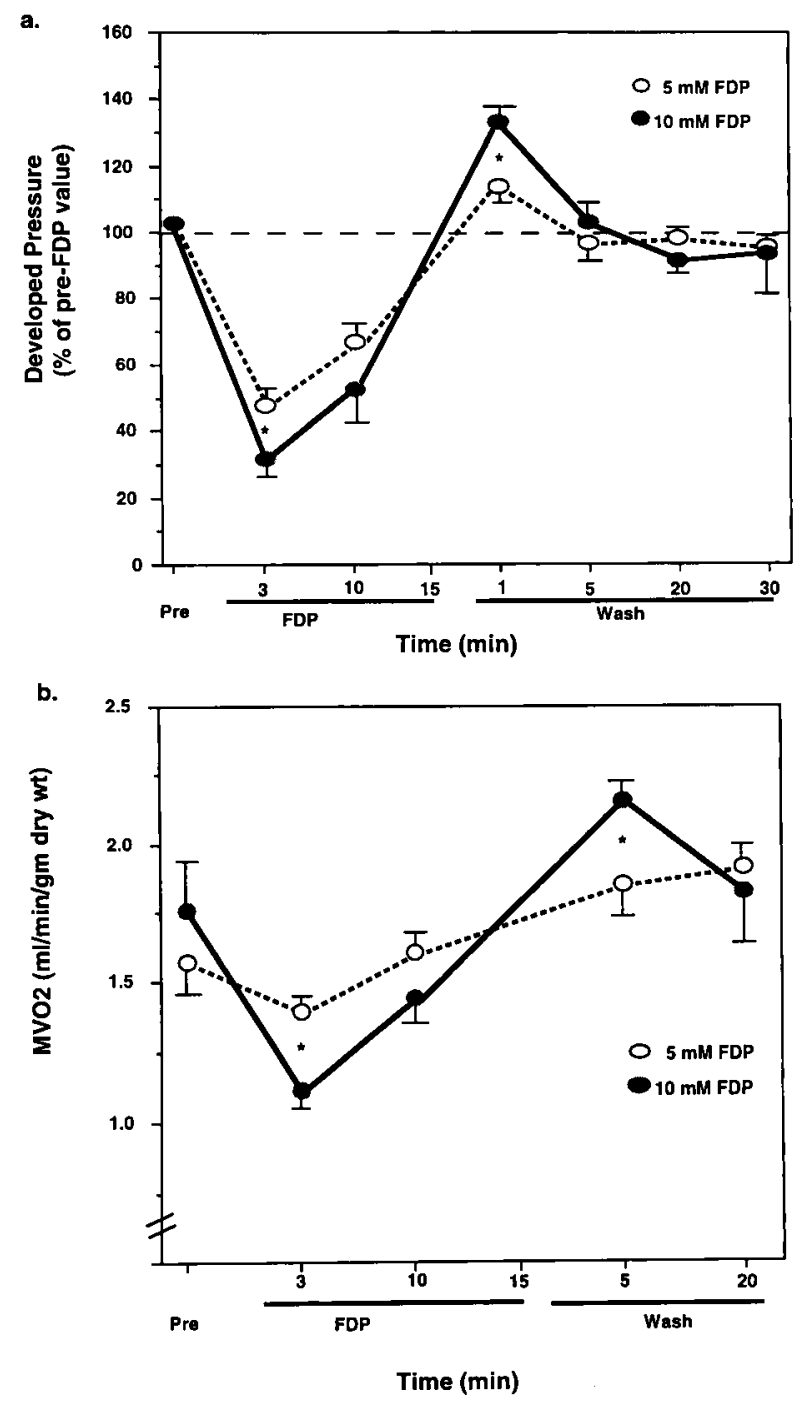

Fig. 2. Effects of FDP infusion on developed pressure (a) and $\mathrm{MVO}_{2}$ (b) followed by Krebs buffer perfusion (wash) in a nonischemic heart model. Developed pressure is expressed as percent of pretreatment (pre) values. All values are mean \pm standard error. ${ }^{*} p<0.05$ compared with pre-FDP values; $n=5$ per group.

several potential mechanisms. Markov and associates $^{4}$ have shown that FDP can restore the depressed glycolytic activity in ischemic myocardium by regulatory effects on the glycolytic pathway and also as a substrate for this pathway. Other investigators have also suggested that FDP can enter the cytosol in sufficient quantities to function as a direct substrate for glycolysis. ${ }^{5-7}$

FDP has also been shown to lower the free $\mathrm{Ca}^{2+}$ concentration in crystalloid solutions such as Krebs- 
a.

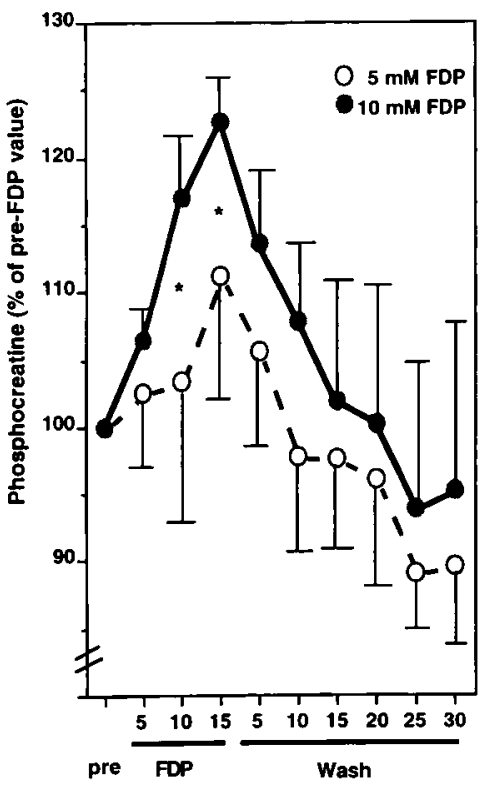

Time (min) b.

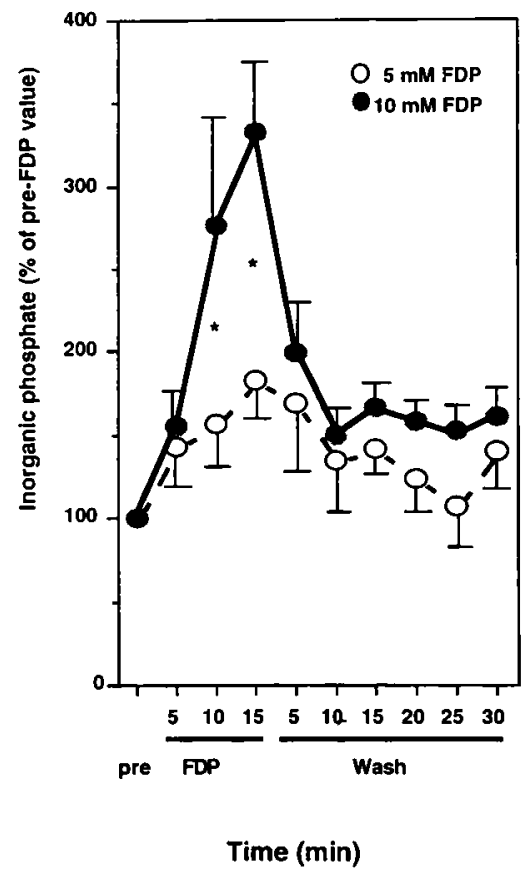

c.

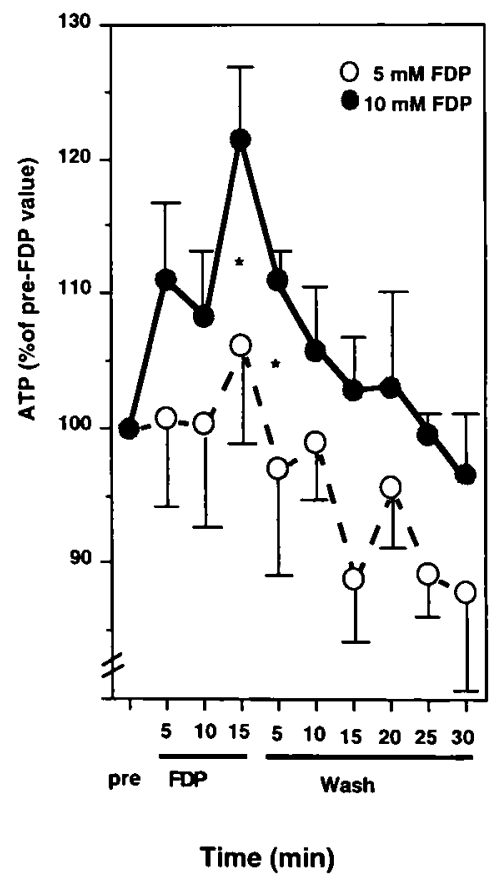

Fig. 3. a, Phosphocreatine; b, inorganic phosphate; c, $\beta$-ATP. Concentrations in perfused nonischemic hearts in response to 5 and $10 \mathrm{mmol} / \mathrm{L}$ concentrations FDP measured by ${ }^{31} \mathrm{P}-\mathrm{NMR}$ spectroscopy. Values are expressed as percent change from pre-FDP (pre) levels. All values are mean \pm standard error. ${ }^{*} p<$ 0.05 compared with pre-FDP values; $n=5$ per group.

Henseleit bicarbonate buffer. $^{7}$ In an isolated rat heart model, FDP was found to chelate extracellular calcium in association with decreased contractile forces. However, the precise relationship between FDP concentration, extracellular ionized calcium levels, and myocardial contractile function has not been fully clarified.

Because glycolysis and extracellular calcium concentration during early reperfusion have been shown to play an important role in recovery of myocardial function, the goals of this study were to determine the effects of FDP on myocardial recovery of contractile function and high-energy phosphates in an isolated rabbit heart model. To further clarify the mechanism of action of FDP on the heart, we studied the effects of FDP on high-energy phosphates, contractile function, and extracellular calcium in a nonischemic isolated crystalloid-perfused rabbit heart preparation.

\section{Materials and methods}

Chemicals and reagents. Chemicals for the Krebs buffer solutions were obtained from Sigma Chemical Co.
(St. Louis, Mo.). FDP was obtained courtesy of Biomedica Foscama (Rome, Italy) and from Sigma Chemical Co.

\section{Nonischemic model}

Animal preparation and experimental protocol. New Zealand White rabbits weighing 3 to $4 \mathrm{~kg}$ were anesthetized with intravenous ketamine hydrochloride $(60 \mathrm{mg} /$ $\mathrm{kg}$ ). The heart was isolated and perfused via an aortic cannula in the Langendorff manner. The perfusate used was Krebs-Henseleit buffer, which contained glucose, 11 $\mathrm{mmol} / \mathrm{L}$, and insulin, $10 \mu / \mathrm{L}$, and was equilibrated with 95\% oxygen and 5\% carbon dioxide and maintained at $37^{\circ} \mathrm{C}$ with the use of water-jacketed glassware. The inferior and superior venae cavae and pulmonary veins were sutured closed. The pulmonary artery was cannulated, and samples were taken anaerobically. Left ventricular pressure was monitored by a pressure transducer connected to a latex balloon inserted in the left ventricle via the left atrium. In the nonischemic heart model, the hearts were perfused for a 30 -minute equilibration period, followed by a 15-minute period of FDP infusion, followed by a washout period with standard Krebs buffer.

$\mathrm{Ca}^{2+}$ titration protocol. To determine the relationship between extracellular FDP, free ionized calcium concentration, and myocardial contractile function, we conducted the following experiment. After an initial 30minute stabilization period, rabbit hearts that had been perfused with Krebs-Henseleit solution were infused with 

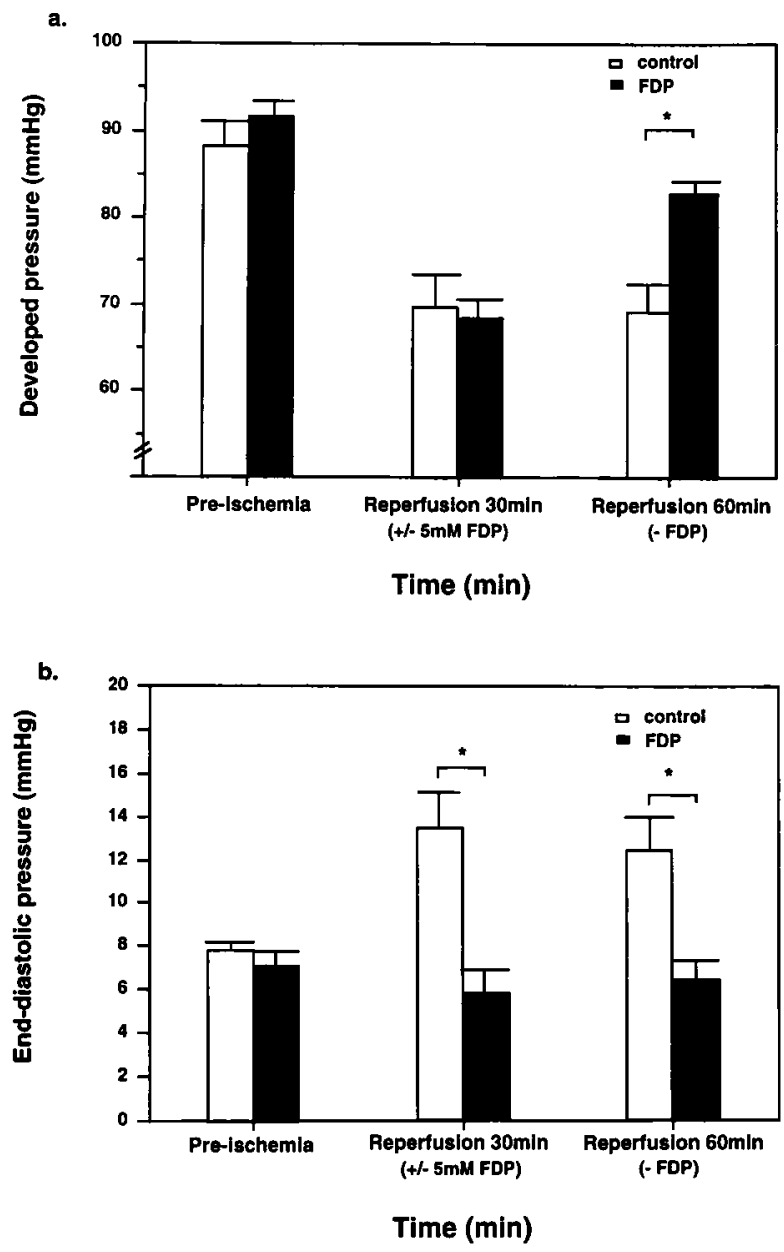

Fig. 4. Developed pressure (a) and end-diastolic pressure (b) in hearts subjected to 30 minutes of ischemia at $37^{\circ} \mathrm{C}$ followed by 60 minutes of reperfusion. In one group, a $5 \mathrm{mmol} / \mathrm{L}$ concentration of FDP was added to the Krebs buffer for the initial 30 minutes of reperfusion followed by 30 minutes with Krebs buffer alone. In a second (control) group, hearts were reperfused with Krebs buffer alone. All values are mean \pm standard error. ${ }^{*} p<0.05$ compared with control hearts; $n=6$ per group.

buffer containing a $5 \mathrm{mmol} / \mathrm{L}$ concentration of FDP. Free ionized calcium in the perfusate was measured with a calcium-sensitive microelectrode (NOVA6, NOVA Medical, Waltham, Mass.), additional calcium $\left(\mathrm{CaCl}_{2}, 0.2\right.$ to $0.8 \mathrm{mmol} / \mathrm{L}$ ) was added to the perfusate buffer, and the final ionized calcium levels were measured. The supplemental $\mathrm{Ca}^{2+}$ infusion was started 1 minute after the onset of the FDP infusion and given for 5 minutes, followed by a 5-minute washout period. Subsequent calcium infusions were given after the 5-minute washout period. Peak developed pressure and ionized calcium concentration of the perfusate were measured at the end of each of the 5-minute calcium infusion periods.
Effects of FDP on high-energy phosphates in the nonischemic heart. Two groups of animals ( $n=5$ per group) were studied. A nonischemic heart model was perfused with Krebs buffer containing FDP (5 and $10 \mathrm{mmol} / \mathrm{L}$ ). Myocardial high-energy phosphates were measured by ${ }^{31} \mathrm{P}-\mathrm{NMR}$ spectroscopy. The hearts were perfused in a $40 \mathrm{~cm}$ horizontal-bore, 4.7 T Bruker BioSpec NMR spectrometer (Bruker Instruments, Inc., Billerica, Mass.) operating at a frequency of $81 \mathrm{MHZ}$. The perfusion chamber was surrounded by a $3.1 \mathrm{~cm}$ diameter, five-turn solenoid coil tuned to $81 \mathrm{MHz}$ for ${ }^{31} \mathrm{P}$ spectroscopy. Ninety-degree radiofrequency pulses were applied with a recycle time of 1 second during a period of 2 minutes 40 seconds, for a total of 148 acquisitions per spectrum. A bulb containing dimethylene phosphonic acid (DMPA) as an internal ${ }^{31} \mathrm{P}$ standard was placed within the right ventricle, and the right atrium was sutured closed. The areas under the $\beta$-adenosine triphosphate (ATP), phosphocreatine (PCr), inorganic phosphate $(\mathrm{Pi})$, and DMPA peaks were determined by integration after baseline correction with the polynomial curve-fitting routine supplied by Bruker Instruments, Inc. All peaks were normalized to the DMPA peaks. Krebs buffer containing FDP (5 and $10 \mathrm{mmol} / \mathrm{L}$ ) was infused for 15 minutes and the hearts were then perfused with plain Krebs buffer for a 30-minute washout period. Developed pressure was monitored continuously during the experiments. Myocardial oxygen consumption $\left(\mathrm{MVO}_{2}\right)$ was measured before FDP infusion, at 3 and 10 minutes of FDP infusion, and after 5 and 20 minutes of washout in both groups.

Ischemia/reperfusion model

Experimental protocol. For the ischemia/reperfusion experiments, the experimental protocol consisted of a 30 minute equilibration period followed by 35 minutes of $37^{\circ} \mathrm{C}$ ischemia induced by aortic clamping, followed by reperfusion. Left ventricular developed pressure and enddiastolic pressure were evaluated before ischemia and at 30 and 60 minutes of reperfusion at the same balloon volume. This duration of ischemia was chosen because we have found that in this animal preparation it produces a significant degree of postischemic contractile dysfunction without the onset of rigor during ischemia. To test for the onset of rigor, after ischemic arrest (approximately 10 to 12 minutes), we adjusted the left ventricular balloon volume to generate a resting left ventricular pressure of 2 $\mathrm{mm} \mathrm{Hg}$ and maintained this pressure until reperfusion. Onset of rigor was defined as a $5 \mathrm{~mm} \mathrm{Hg}$ rise in left ventricular resting pressure. Ischemic rigor was not observed in any of the hearts in these experiments. After reperfusion, the balloon volume was returned to the preischemic level for the contractile function measurements.

In the FDP-treated hearts, a stock solution of FDP adjusted to $\mathrm{pH} 7.4$ with $\mathrm{NaOH}$ and Krebs-Henseleit solution was infused into the aortic cannula at a rate to yield a final concentration of $5 \mathrm{mmol} / \mathrm{L}$ for the first 30 minutes of reperfusion. Reperfusion was then continued with plain Krebs buffer until the end of the experiment. Control hearts received only Krebs buffer during the entire reperfusion period.

Metabolic analysis. $\mathrm{MVO}_{2}$, glucose extraction, and lactate production were assessed before ischemia and at 2.5, 
Table I. Diastolic and developed pressure in the ischemia/reperfusion model

\begin{tabular}{|c|c|c|c|c|c|c|}
\hline & \multicolumn{3}{|c|}{ Diastolic pressure $(\mathrm{mm} \mathrm{Hg})$} & \multicolumn{3}{|c|}{ Developed pressure $(\mathrm{mm} \mathrm{Hg})$} \\
\hline & \multirow{2}{*}{$\begin{array}{c}\text { Pre- } \\
\text { ischemia }\end{array}$} & \multicolumn{2}{|c|}{ Reperfusion } & \multirow{2}{*}{$\begin{array}{c}\text { Pre- } \\
\text { ischemia }\end{array}$} & \multicolumn{2}{|c|}{ Reperfusion } \\
\hline & & $30 \mathrm{~min}$ & $60 \mathrm{~min}$ & & $30 \mathrm{~min}$ & $60 \mathrm{~min}$ \\
\hline Control & $8 \pm 0.4$ & $19 \pm 2$ & $17 \pm 2$ & $92 \pm 3$ & $74 \pm 3$ & $77 \pm 2$ \\
\hline FDP $(5 \mathrm{mmol} / \mathrm{L})$ & $7 \pm 0.2$ & $14 \pm 3$ & $9 \pm 2^{*}$ & $95 \pm 1$ & $80 \pm 4$ & $94 \pm 1^{*}$ \\
\hline $\mathrm{FDP}(5 \mathrm{mmol} / \mathrm{L})+\mathrm{Ca}$ & $8 \pm 0.5$ & $17 \pm 2$ & $12 \pm 1^{*}$ & $96 \pm 1$ & $81 \pm 3$ & $90 \pm 1^{*}$ \\
\hline
\end{tabular}

* $p<0.05$ versus control; $n=4$ per group.

$5,10,30,32.5$, and 45 minutes of reperfusion. $\mathrm{MVO}_{2}$ was derived from the difference of oxygen tension between aortic perfusate and coronary effluent (ABL-3 Acid-Base Laboratory, Radiometer A/S, Copenhagen, Denmark) and the coronary flow rate. Glucose extraction was derived from the difference in glucose concentration between aortic perfusate and coronary effluent, multiplied by coronary flow, and divided by heart weight. Lactate released from the heart into the coronary was measured by enzymatic analysis (lactate-glucose analyzer 2300 STAT, YSI, Yellow Springs, Ohio) and determined by the calculation of coronary flow times lactate per gram dry weight.

High-energy phosphatemeasurements. High-energy phosphate content was measured in an additional two groups of animals ( $n=6$ per group) subjected to 35 minutes of normothermic ischemia followed by reperfusion. In one group a $5 \mathrm{mmol} / \mathrm{L}$ concentration of FDP was infused during the first 30 minutes of reperfusion followed by 15 minutes of washout with plain Krebs buffer. The second group was subjected to the same ischemic injury but was reperfused only with Krebs buffer for 45 minutes and served as the control. Details of the NMR measurements were the same as described earlier (nonischemic model).

All animals received humane care in compliance with the "Principles of Laboratory Animal Care" formulated by the National Society for Medical Research and the "Guide for the Care and Use of Laboratory Animals" prepared by the Institute of Laboratory Animal Resources and published by the National Institutes of Health (NIH Publication No. 86-23, revised 1985). This protocol was reviewed and approved by the animal care committee at Children's Hospital.

Statistical analysis. Analysis of variance was used for comparisons of multiple data points between two groups. When significant differences were detected by analysis of variance, the unpaired $t$ test was used to assess differences between individual time points within or between the groups. All data are expressed as the mean \pm standard error of the mean.

\section{Results}

\section{Nonischemic model}

$\mathrm{Ca}^{2+}$ titration experiments. Fig. 1, a, depicts the ionized $\mathrm{Ca}^{2+}$ concentration in the perfusate containing a $5 \mathrm{mmol} / \mathrm{L}$ concentration of FDP plotted against the additional $\mathrm{CaCl}_{2}$. The $5 \mathrm{mmol} / \mathrm{L}$ FDP concentration significantly decreased the $\mathrm{Ca}^{2+}$ con- centration of Krebs buffer from $1.19 \pm 0.03 \mathrm{mg} / \mathrm{dl}$ to $0.81 \pm 0.02 \mathrm{mg} / \mathrm{dl}$ (31.8\% decrease). With the additional $\mathrm{CaCl}_{2}, \mathrm{Ca}^{2+}$ increased linearly so that an additional $0.6 \mathrm{mmol} / \mathrm{L}$ total calcium concentration was required to return the ionized calcium concentration to pre-FDP levels. Fig. $1, b$, shows, in an isolated perfused heart model, the change in developed pressure versus ionized calcium concentration obtained with the titration scheme described above. Despite the additional calcium to correct for the calcium-binding effects of FDP, developed pressure did not recover fully during the FDP infusion in these hearts, suggesting that the effect of FDP on contractile function is only partly related to extracellular calcium levels.

Contractile function and metabolic effects of FDP. The nonischemic hearts receiving a $5 \mathrm{mmol} / \mathrm{L}$ concentration of FDP had a fall in developed pressure from $69 \pm 6 \mathrm{~mm} \mathrm{Hg}$ to $31 \pm 5 \mathrm{~mm} \mathrm{Hg}$ ( $45 \%$ of basal value). In hearts perfused with a $10 \mathrm{mmol} / \mathrm{L}$ concentration of FDP, developed pressure fell from $75 \pm 4 \mathrm{~mm} \mathrm{Hg}$ to $23 \pm 3 \mathrm{~mm} \mathrm{Hg}(31 \%$ of basal value) with a gradual recovery during the FDP infusion in both groups (Fig. 2,a). On washout of the FDP, however, developed pressure immediately recovered to $79 \pm 8 \mathrm{~mm} \mathrm{Hg}$ (114\% of basal value) and $104 \pm$ $4 \mathrm{~mm} \mathrm{Hg}$ (133\% of basal value) in the $5 \mathrm{mmol} / \mathrm{L}$ and 10 mol/L FDP groups, respectively, followed by a gradual return to baseline values. Fig. 2, $b$, depicts the $\mathrm{MVO}_{2}$ during the FDP infusion and washout period, with a significant decline during FDP infusion followed by recovery during washout.

High-energy phosphates. The PCr level rose significantly during the FDP infusion, with the rise being greater with a $10 \mathrm{mmol} / \mathrm{L}$ concentration of FDP (Fig. 3, a). Similarly, the ATP concentration rose during FDP infusion, with a greater accumulation in the hearts receiving a $10 \mathrm{mmol} / \mathrm{L}$ concentration of FDP (Fig. 3, c). Interestingly, the level of Pi also rose most dramatically during the $10 \mathrm{mmol} / \mathrm{L}$ FDP infusion, with nearly complete recovery by $10 \mathrm{~min}$ utes of washout (Fig. 3,b). The marked elevation in Pi occurred despite the rise of high-energy phosphates, indicating a net accumulation of phosphate within the cell during the FDP infusion. 
a.

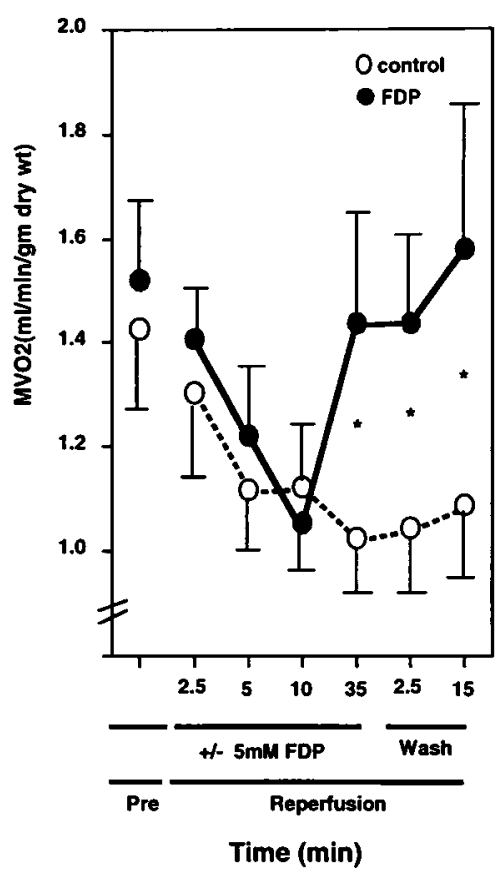

b.

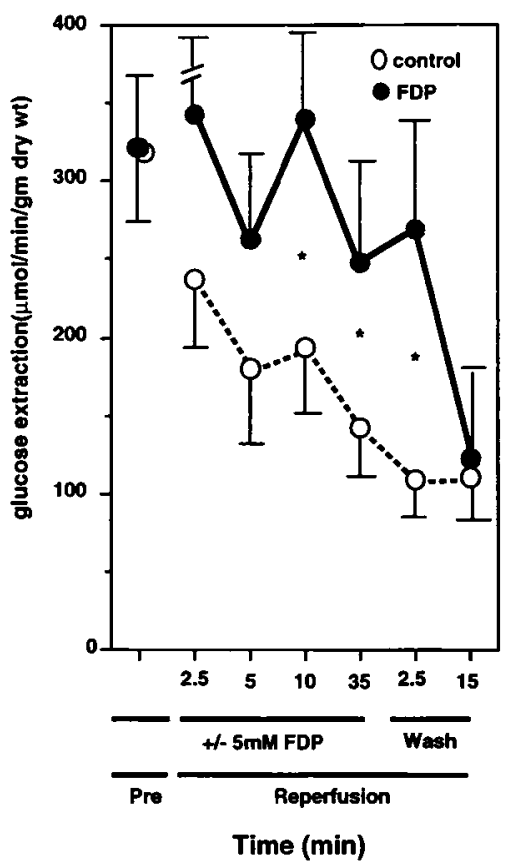

c.

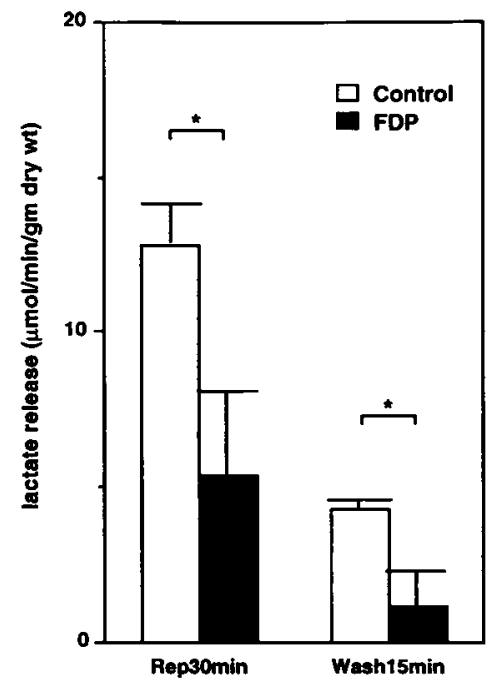

Fig. 5. $\mathrm{MVO}_{2}$ (a), glucose uptake (b), and lactate release (c) in hearts after 30 minutes of $37^{\circ} \mathrm{C}$ ischemia and 45 minutes of reperfusion. In one group, a $5 \mathrm{mmol} / \mathrm{L}$ concentration of FDP was added to the Krebs buffer for the initial 30 minutes of reperfusion followed by 15 minutes with Krebs buffer alone (wash). In a second (control) group, hearts were reperfused with Krebs buffer alone. Lactate release represents the total lactate released into the coronary effluent during the indicated reperfusion period. All values are mean \pm standard error. ${ }^{*} p<0.05$ compared with control hearts; $n=6$ per group.

\section{Ischemia/reperfusion model}

Contractile function. Left ventricular resting pressure measured at constant balloon volume did not change during the 35-minute ischemic period in any of the hearts studied, indicating that this degree of ischemia did not lead to ischemic contracture.

After 35 minutes of normothermic ischemia and 30 minutes of reperfusion, left ventricular developed pressure decreased from $88 \pm 3 \mathrm{~mm} \mathrm{Hg}$ to $70 \pm 4$ $\mathrm{mm} \mathrm{Hg}(78 \%$ of preischemic level) in control untreated hearts and from $92 \pm 2 \mathrm{~mm} \mathrm{Hg}$ to $68 \pm 3$ $\mathrm{mm} \mathrm{Hg} \mathrm{(74 \%} \mathrm{of} \mathrm{preischemic} \mathrm{level)} \mathrm{in} \mathrm{the} \mathrm{FDP-}$ treated hearts. On further reperfusion with Krebs buffer (without FDP) in both groups, there was significantly better recovery of developed pressure in the FDP-treated hearts (91\% of preischemic level) by 60 minutes of reperfusion (Fig. 4, $a$ ) compared with the control untreated hearts. Diastolic pressure was significantly elevated by $30 \mathrm{~min}$ utes of reperfusion in control hearts (from $7.8 \pm 0.5$ $\mathrm{mm} \mathrm{Hg}$ before ischemia to $13 \pm 2 \mathrm{~mm} \mathrm{Hg}$ ) without further recovery by 60 minutes of reperfusion. In contrast, in the FDP-treated hearts, diastolic pressure remained at preischemic values during the entire reperfusion period (Fig. $4, b$ ).

To determine whether the beneficial effects of FDP on reperfusion were due to its ability to bind calcium and thus lower extracellular calcium content during early reperfusion, we performed the following experiments. In a separate group of hearts ( $n=4$ per group), the same experiment as described above was repeated, but this time ionized calcium was normalized $(1.2 \mathrm{mmol} / \mathrm{L})$ in the perfusate containing FDP $(5 \mathrm{mmol} / \mathrm{L})$. Hearts were reperfused with Krebs buffer containing FDP with or without calcium during the first 30 minutes of reperfusion followed by an additional 30 minutes of perfusion with Krebs buffer (without FDP). Diastolic and developed pressure was measured after reperfusion and compared with pressures in a group receiving FDP without additional calcium and a control group receiving only Krebs buffer. Recovery of developed pressure was significantly better in the FDP-treated hearts than 


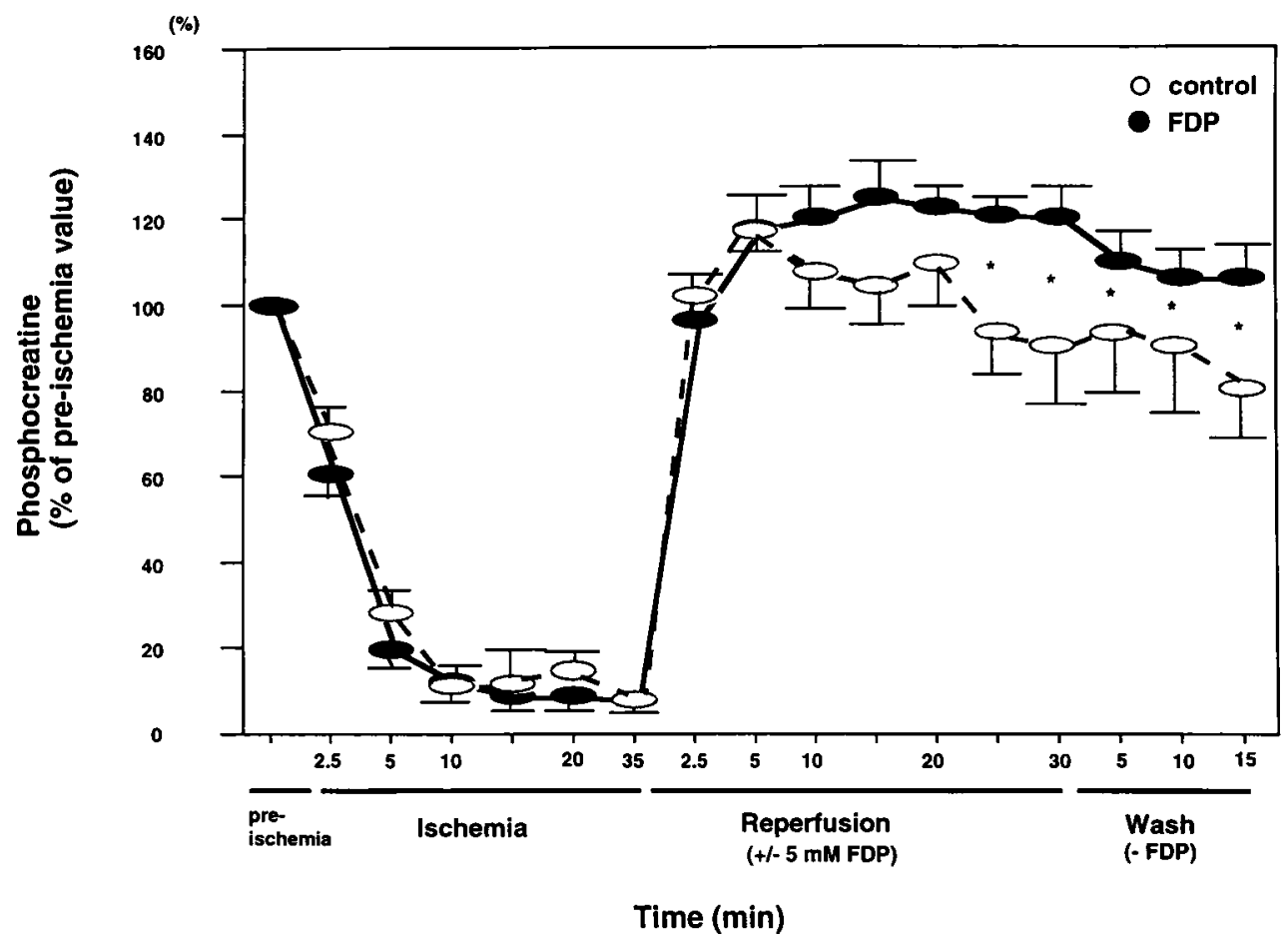

Fig. 6. Phosphocreatine concentration (expressed as percent of preischemic value) during ischemia and 45 minutes of reperfusion. In one group, a $5 \mathrm{mmol} / \mathrm{L}$ concentration of FDP was added to the Krebs buffer for the initial 30 minutes of reperfusion followed by 15 minutes of perfusion with Krebs buffer alone (wash). In a second (control) group, hearts were reperfused with Krebs buffer alone. All values are mean \pm standard error. ${ }^{*} p<0.05$ compared with control hearts; $n=6$ per group).

in controls, with no difference between the FDP plus calcium group versus the FDP without added calcium group (Table I). Similarly, diastolic pressure was significantly lower in both FDP-treated groups than in control hearts, with no difference between the two FDP-treated groups.

Substrate use. Fig. 5, a, depicts $\mathrm{MVO}_{2}$ before ischemia and during reperfusion. In both control and FDP-treated hearts, $\mathrm{MVO}_{2}$ was significantly lower than preischemic levels during the first 10 minutes of reperfusion. In FDP-treated hearts, however, $\mathrm{MVO}_{2}$ recovered to preischemic levels by the end of the FDP infusion (30 minutes of reperfusion), and $\mathrm{MVO}_{2}$ was maintained at those levels during the subsequent 15 minutes of plain Krebs perfusion. In control hearts, $\mathrm{MVO}_{2}$ remained depressed during the entire reperfusion period. Glucose extraction was also maintained at preischemic levels in the FDP-treated hearts with a decline on FDP withdrawal. The higher glucose uptake on reperfusion in the FDP-treated hearts was associ- ated with significantly lower lactate release from these hearts, indicating increased oxidative metabolism compared with control untreated hearts (Fig. 5, $b$ and $c$ ).

Myocardial high-energy phosphates. PCr levels rapidly fell after the onset of ischemia followed by a more gradual decline in ATP levels during ischemia (Fig. 6 and Table II). On reperfusion there was a rapid recovery in $\mathrm{PCr}$ with a typical overshoot seen in the first 5 minutes of reperfusion. In FDP-treated hearts, supernormal PCr levels were maintained during the FDP infusion, with gradual return to preischemic levels during the washout period. In control hearts, after the initial overshoot, $\mathrm{PCr}$ levels fell to levels slightly below preischemic values by the end of the 45-minute reperfusion period. Similar to the FDP-treated nonischemic hearts, postischemic FDP-treated hearts had higher levels of intracellular Pi during the FDP infusion. However, Pi concentration recovered to control levels after FDP withdrawal (Table II). 
Table II. Effect of FDP on Pi and ATP in the ischemia/reperfusion model

\begin{tabular}{lcccc}
\hline & & \multicolumn{3}{c}{30 min } \\
& $\begin{array}{c}\text { Pre- } \\
\text { ischemia } \\
(\%)\end{array}$ & $\begin{array}{c}\text { End- } \\
\text { ischemia } \\
( \pm 5 \text { mmol/L } \\
\text { FDP })\end{array}$ & $\begin{array}{c}15 \text { min } \\
\text { wash } \\
(-F D P)\end{array}$ \\
\hline Pi & & & & \\
Control & 100 & $854 \pm 80$ & $223 \pm 62$ & $282 \pm 77$ \\
FDP (5 mmol/L) & 100 & $734 \pm 103$ & $386 \pm 82^{*}$ & $288 \pm 63$ \\
ATP & & & & \\
Control & 100 & $65 \pm 5$ & $72 \pm 4$ & $59 \pm 4$ \\
FDP (5 mmol/L) & 100 & $56 \pm 5$ & $78 \pm 5$ & $66 \pm 4$
\end{tabular}

Values are expressed as percent of preischemic values.

${ }^{*} p<0.05$ versus control; $n=6$ per group.

\section{Discussion}

This study demonstrates that FDP has important dose-dependent effects on the crystalloid-perfused nonischemic heart, including increased glucose uptake and high-energy phosphate levels. Exogenous FDP also caused a fall in developed pressure, owing in part to its ability to bind free calcium and in part to elevated intracellular Pi levels. ${ }^{8}$ In the postischemic heart, FDP given during early reperfusion leads to significantly improved recovery of systolic and diastolic function in association with increased glucose uptake and PCr levels.

FDP is an intermediate in the glycolytic pathway, which is formed by the phosphorylation of fructose- 6 phosphate and is cleaved into dihydroxyacetone phosphate and glyceraldehyde 3-phosphate by aldolase. Conversely, FDP can be converted to fructose- 6 phosphate by hydrolysis of the phosphate ester at $\mathrm{C}-1$ during gluconeogenesis. Under physiologic conditions, FDP levels in the cytosol are maintained in the micromolar range. Our finding that a high extracellular FDP concentration was associated with increased glucose uptake in both the nonischemic and postischemic hearts indicates that one of the effects of exogenous FDP is to accelerate glycolytic flux in the cell. There are two proposed mechanisms to explain the increased glucose uptake seen with exogenous FDP. One explanation is that FDP can enter the cytosol and provide a direct substrate for the glycolytic pathway entering at an energetically favorable level where ATP-dependent phosphorylation is not required. The second explanation is that FDP acts to stimulate glycolysis by increasing glycolytic or Krebs cycle enzyme activity leading to increased glycolytic flux and oxidative metabolism. 3, 7, 9-11

Phosphorylation of sugars has been generally regarded as a mechanism for trapping these substrates inside cells owing to the low permeability through biologic membranes of the sugar phosphate ester. Even Pi translocation across the plasma membrane has been shown to be slow. ${ }^{9}$ However, since the concentration of free FDP in the cytosol is only $1.2 \mu \mathrm{mol} / \mathrm{L},{ }^{6}$ an infusion of FDP in the millimolar concentration range results in a more than thousand-fold gradient across the plasma membrane. The fact that, in our study, the effects of FDP in the heart were dose dependent and that total intracellular phosphates were increased during FDP infusion further supports the contention that FDP can cross the sarcolemmal membrane under these experimental conditions. Other investigators studying the effects of exogenous FDP in hypoxic smooth muscle cells have provided direct evidence that FDP can cross the cell membrane and serve as a metabolic substrate. ${ }^{3}$ Using ${ }^{13} \mathrm{C}$-labeled FDP, they demonstrated that FDP enters the cell and leads to the production of ${ }^{13} \mathrm{C}$-lactate during hypoxia in a dosedependent manner. The mechanism of entry of FDP into the cells is not known. However, owing to structural similarities between FDP and membrane phospholipids, investigators have suggested that FDP can interact with membrane proteins to create polar discontinuities that may permit FDP to permeate the cell membrane. ${ }^{10}$

The exact effects of exogenous FDP on glucose metabolism inside the cell are not completely clear. Hassinen and associates ${ }^{7}$ have suggested that external FDP is not used as a glycolytic substrate in the heart, since they found that maximal protection from anoxic damage was achieved with a $6 \mathrm{mmol} / \mathrm{L}$ concentration of FDP only in the presence of glucose, suggesting a pharmacologic effect of FDP on glycolytic enzyme activity. Similar results have been shown in astrocytes subjected to hypoxia. ${ }^{11}$ Glycolytic activity is regulated not only by substrate availability but also by enzyme activity, particularly hexokinase, phosphofructokinase, and pyruvate kinase. Hexokinase, phosphofructokinase, and pyruvate kinase are believed to have regulatory as well as catalytic roles in glycolysis. ${ }^{12} \mathrm{FDP}$, a product of the early steps in glycolysis, activates pyruvate kinase to enable it to keep pace with the oncoming high flux of intermediates. Exogenous FDP may therefore be able to activate pyruvate kinase and cause acceleration of glycolysis. ${ }^{13}$ Alternatively, reversal of the glycolytic steps producing fructose-6-phosphate may lead to fructose 2,6-bisphosphate generation using the enzyme fructose 1,6-bisphosphatase. ${ }^{2}$ Fructose 
2,6-bisphosphate is a known potent activator of phosphofructokinase and serves to stimulate glycolytic flux. ${ }^{13,14}$

The mechanism by which exogenous FDP decreases contractile force in the nonischemic heart is probably multifactorial. Our observation that a 5 $\mathrm{mmol} / \mathrm{L}$ concentration of FDP is able to lower free $\mathrm{Ca}^{2+}$ concentration by $32 \%$ in standard Krebs buffer and that by normalizing ionized calcium most of the contractile function returns indicates that this is the major mechanism of FDP effects on myocardial contractility. Hassinen and associates ${ }^{7}$ showed a similar effect of FDP on contractile function in an isolated rat heart model and also ascribed the effects to calcium chelation. Although the negative inotropic effects of FDP on the normal nonischemic heart may seem undesirable, during early reperfusion the calcium chelating effects of FDP may provide some protection. Calcium overload during reperfusion is a widely known mechanism of injury to the heart after severe reversible ischemia, and the ability of FDP to chelate calcium may explain some of the beneficial effects we observed in our study. Whether FDP prevents calcium overload simply by chelating extracellular calcium or whether it is capable of entering the cell to lower calcium we cannot determine in this study, because we did not measure intracellular calcium levels. However, in the experiments in which hearts were reperfused with FDP and the ionized calcium was normalized $(1.2 \mathrm{mmol} / \mathrm{L})$, we were unable to detect any significant difference in recovery of contractile function compared with the hearts receiving FDP alone. These results indicate that, in our model of ischemia/reperfusion, the calcium-chelating activity of FDP is not a major mechanism for the improved recovery of postischemic contractile function.

Regardless of the exact mechanism, however, our study demonstrates that exogenous FDP, when given during early reperfusion, increases glucose uptake, oxidative metabolism, and PCr levels leading to significantly improved recovery of contractile function in the postischemic crystalloid-perfused rabbit heart.
We gratefully acknowledge Miss Mary Ann Butowicz for her technical assistance in the performance of this project.

\section{REFERENCES}

1. Grandl AM, Muggia C, Barzizza F, Venco A, Finardi G. Improved left ventricular function after short-term treatment with fructose-1,6-diphosphate: echocardiographic study in chronic ischemic heart disease and idiopathic dilated cardiomyopathy. Clin Ther 1988;10:372-80.

2. Marchionni N, Conti A, De Alfieri W, et al. Hemodynamic and electrocardiographic effects of fructose 1,6-diphosphate in acute myocardial infarction. Am J Cardiol 1985;56:266-9.

3. Hardin CD, Roberts TM. Metabolism of exogenously applied fructose 1,6-bisphosphate in hypoxic vascular smooth muscle. Am J Physiol 1994;267:H2325-32.

4. Markov AK, Oglethorpe NC, Blake TM, Lehan PH, Hellems HK. Hemodynamic, electrocardiographic, and metabolic effects of fructose diphosphate on acute myocardial ischemia. Am Heart J 1980;100:639-46.

5. Nuutinen EM, Lazzarino G, Giardina B, Hassinen IE. Effect of exogenous fructose 1,6-bisphosphate glycolysis in the isolated perfused rat heart. Am Heart J 1991;122:523-7.

6. Lazzarino G, Nuutinen ME, Tavazzi B, Cerroni D, Di Pierro D, Giardina B. Preserving effect of fructose-1,6-bisphosphate on high-energy phosphate compounds during anoxia and reperfusion in isolated Langendorff-perfused rat hearts. J Moll Cell Cardiol 1991;23:13-23.

7. Hassinen IE, Nuutinen EM, Ito K, et al. Mechanism of the effect of exogenous fructose 1,6-bisphosphate on myocardial energy metabolism. Circulation 1991;83:584-93.

8. Kentish J. The effects of inorganic phosphate and creatine phosphate on force production in skinned muscle from rat ventricle. J Physiol (Lond) 1986;370:585-604.

9. Medina G, Illingworth J. Some factors affecting phosphate transport in a perfused rat heart preparation. Biochem J 1980;188:297-311.

10. Galzinga L, Rizzoli V, Bianchi M, Rigobello MP, Scuri R. Some effects of fructose 1,6-diphosphate on rat myocardial tissue related to membrane stabilizing action. Cell Biochem Funct 1989;7:91-6.

11. Gregory GA, Yu ACH, Chan PH. Fructose 1,6-bisphosphate protects astrocytes from hypoxic damage. J Cereb Blood Flow Metab 1989;9:29-34.

12. Stryer L. Glycolysis. Stryer biochemistry. 3rd ed. New York. WH Freeman: 1988. p. 350-72.

13. Taylor CB, Bailey E. Activation of liver pyruvate kinase by fructose 1,6-diphosphate. Biochem J 1967;102:32-3.

14. Depre C, Ride MH, Veitch K, Hue L. Role of fructose 2,6-bisphosphate in the control of heart glycolysis. J Biol Chem 1993;268:13274-9.

15. Hue L, Rider MH. Role of fructose 2,6-bisphosphate in the control of glycolysis in mammalian tissues. Biochem J 1987; 245:313-24. 